\title{
Comparative analysis of high average-utility patterns algorithms
}

\author{
C.Sivamathi ${ }^{* *}$ and S.Vijayarani ${ }^{2}$ \\ Research Scholar, Department of Computer Science, Bharathiar University, Tamil Nadu, India ${ }^{1}$ \\ Assistant Professor, Department of Computer Science, Bharathiar University, Tamil Nadu, India ${ }^{2}$
}

C2018 ACCENTS

\begin{abstract}
Utility mining is a current emerging field in data mining. Utility mining raises various forms. They are high utility itemset mining, utility frequent itemset mining, negative utility itemsets mining, rare high utility itemset mining etc. All these itemset mining does not consider the size of itemsets. A recent development in utility mining is high average-utility itemset mining. This average utility mining considers length of itemsets along with the utility of itemsets. There are several algorithms proposed to retrieve high average utility itemsets in a database. The main objective of this work is to compare three high average-utility patterns algorithms: high average-utility patterns (HAUP) algorithm, high average-utility itemset-miner (HAUI-Miner) algorithm and efficient high average-utility pattern mining (EHAUPM) algorithm. The execution time and memory space are considered as performance measures for the comparison. It was found that EHAUPM algorithm performs better than other algorithms.
\end{abstract}

\section{Keywords}

Utility mining, High average utility mining, Average high utility itemsets, Length of itemsets, HAUP algorithm, HAUIminer algorithm, EHAUPM algorithm.

\section{Introduction}

Utility mining picks up highly utility itemsets in a database [1]. The term utility can be any significant measure of an item based on the nature of domain [2, 3]. Utility mining incorporates various forms like high utility itemset/patterns mining, utility frequent itemset mining, negative utility itemsets mining, rare high utility itemset mining, sequence high utility mining, high utility association mining etc. In all these forms, the main objective is to retrieve high utility itemsets (HUI) based on minimum threshold value. The patterns with utility value greater than threshold are known as HUI. All these forms do not consider the length of the patterns. This is considered as drawback in traditional utility mining algorithms $[4,5]$, since length of patterns is also has major impact on utility of patterns. The utilities of itemsets be likely to have more utility for itemsets, that has more items [4-6]. For example utility of two itemset is less than the utility of three itemsets. To overcome this issue, high average utility itemset mining was proposed. It considers both length and utility of itemset. Hence it is a more fair measurement of the utilities of itemsets for real-life applications.

*Author for correspondence

276
Traditional utility mining algorithms calculate utility value of a pattern as the product of its internal and external utility value. Internal utility of a pattern is any significant measure of a pattern defined by the expert of the field. External utility of the pattern is a unit measure of the pattern. Several utility measures are defined in traditional utility mining algorithms to retrieve high utility patterns from a database [4-8]. They are Transaction Utility, Transaction weighted utility, High transaction weighted utility itemsets, utility of itemset [1-4]. All these measures consider only the utility values of the patterns, not the length of patterns. The length of patterns increases, the utility of patterns also increases. Hence high average utility itemset mining (HAUIM) was proposed. The average utility is defined as the sum of the utilities of the itemset in transactions where it appears, divided by the number of items that it contains. This measure overcomes the drawback traditional utility mining algorithms.

The main objective of this work is to compare three High average-utility patterns algorithms HAUP algorithm, HAUI-miner algorithm and EHAUPM algorithm. 


\section{Preliminaries in high average utility patterns}

Let $I=\{i 1, i 2, i 3 \ldots \ldots \ldots \ldots i m\}$ be a set of items. Let $\mathrm{D}=\{\mathrm{T} 1, \mathrm{~T} 2, \mathrm{~T} 3 \ldots . . \mathrm{Tn}\}$ be a database where $\mathrm{Ti}$ represents transactions. Let $\mathrm{P}=\{\mathrm{P}(\mathrm{i} 1), \quad \mathrm{P}(\mathrm{i} 2)$ .........p $(\mathrm{im})\}$ be an unit of items i. Let $\mathrm{X}$ be an itemset contain items im and let $\mathrm{k}$ be the length of itemset. The length of itemset is the number of items in $\mathrm{X}$. Let $\mu$ be a minimum average-utility threshold. Preliminary definitions for calculating utility values of itemsets are as follows [1-5]:

Definition 1: The internal utility of an item is quantity of item in a transaction database.

Definition 2: The external utility of an item is a unit profit of an item.

Definition 3: Utility function $\mathrm{f}$ is the product of internal and external utility

Definition 4: The utility of item in transaction $\mathrm{T}$ is the utility function of the item in that particular transaction.

Definition 5: The utility of itemset $\mathrm{S}$ in transaction $\mathrm{T}$ is defined sum of utility of items in that itemset in a transaction.

Definition 6: The utility of itemset $\mathrm{S}$ in database DB is defined as the sum of utility of itemsets in all transactions.

Definition 7: The utility of transaction $\mathrm{T}$ is defined as the sum of utility of each item in a transaction.

Definition 8: Itemset $\mathrm{X}$ is said to be high utility itemset if and only if its utility is greater than minimum utility threshold.

\section{Related works}

Krishnamoorthy [3] proposed the two-phase algorithm for HUI mining. In the first phase, database is scanned and generated candidates are pruned by using the upper bound threshold. In next phase, database is scanned again, the actual utility value of remain candidates are calculated and the algorithm then gets HUI from candidates. The problem of this method is to reduce candidates and time for rescanning database.

In [4], the authors compared high utility algorithms. The authors analyzed two-phase, UP-growth+, efficient fast itemset mining (EFIM) algorithm, fast high utility mining (FHM) algorithm, high utility itemset miner (HUI-Miner) algorithm, direct discovery of high utility pattern (D2HUP) algorithms. In this work, execution time and memory space occupied by the algorithms are considered as performance measures. In terms of execution time, D2HUP was better, which executed fastly than the other one. By memory space, HUI-Miner occupies less space than the remaining ones.

Almost all algorithms in literature extract HAUI using a single common minimum utility threshold. In [5] the authors introduced the concept of multiple minimum average utility thresholds. In this work, each item has different thresholds. Also, improved estimated utility co-occurrence pruning strategy (IEUCP) and pruning before calculation strategy (PBCS) strategies are introduced to prune the itemsets. In [6], the authors described various forms of utility mining like negative utility mining, on shelf utility mining, frequent utility mining concepts, high average utility itemset mining, closed high utility itemset mining etc. The authors also explained utility calculations with neat example.

Most of utility mining algorithms can handle static databases. But in real-world applications, dynamic databases are used. These databases are modified dynamically by inserting new transactions. There are some algorithms available to retrieve HUI from dynamic databases. Such algorithms are termed as incremental high-utility itemset mining (IHUIM) algorithms. In [8] the authors provide a detailed survey of IHUIM algorithms.

In Hong et al. [9] used average utility upper-bound to prune generated candidates and create the set of itemsets having upper-bound value are greater than or equal threshold. The set of $(\mathrm{r}+1)$-itemset is generated by combining r-itemsets and 1- itemsets which have high average utility upper-bound. In [10] the authors proposed EHAUPM algorithm. To improve the performance of HAUIM, this paper proposes two novel tighter upper-bound models as alternative to the traditional average-utility upper-bound (AUUB) model for mining HAUIs. The looser upper-bound model (LUB) considers the remaining-maximum utility in transactions to reduce the upper-bound on the utilities of itemsets. The second upper-bound model ignores irrelevant items in transactions to further tighten the upper-bound. Three pruning strategies are also designed.

In [11], the authors proposed, HAUI-Miner algorithm. They presented an efficient average-utility (AU)-list structure to discover the high average utility itemsets more efficiently. The algorithm explores the search space without candidate generation. Also an efficient pruning strategy was implemented to reduce the search space and speed up the mining process. Hong et al. [9] proposed an algorithm to reveal 
HAUIs. It was a projection based level-wise algorithm. The authors also proposed a new upperbound model based on prefix concept. This reduces the number of unpromising candidates when compared to the TWU model.

\section{Existing algorithms}

4.1High average utility itemset using UP tree (HAUP tree) algorithm:

Lin et al. [12] proposed a tree called high average utility pattern tree (HAUP tree) structure is to store utility information of itemsets. The HAUP-growth algorithm is proposed to mine high average utility itemsets from this tree structure. This algorithm integrates the high average-utility mining algorithm and the FP-tree-like approach. The algorithm proposed a condensed tree structure for efficiently deriving high average utility patterns. The tree structure is called the HAUP tree [12]. Each node at the end of a path in the tree has to store the averageutility upper bound of the item in the node as well as the quantities of its preceding items in the path. Once the tree was constructed, Process the items in the Header_Table one by one from bottom. All the nodes with the currently processed item in the HAUP tree are combined to form the itemsets. If an itemset is generated from more than one path, merge the quantities from the two arrays by addition. Then the actual average-utility value of each merged itemset is calculated. Now verify whether the actual averageutility value of each generated average utility itemset is larger than or equal to the minimum average-utility value. If it is, then the items are high average-utility itemsets.

\subsection{High average-utility itemset- miner (HAUI-} Miner) algorithm

HAUI-Miner algorithm uses an average-utility (AU)list structure to store the information needed by the mining process. Moreover, an algorithm named HAUI-Miner is also developed to mine HAUIs more efficiently than previous works [12]. In this work, transaction-maximum utility downward closure (TMUDC) property was introduced. This effectively reduces the search space [12], and hence allows pruning unpromising candidates early. Hence this algorithm reduces the search space to efficiently discover the actual high average utility patterns [11, 12]. The transaction-maximum utility of a transaction is defined as the maximum utility of items in a transaction. The average-utility upper-bound of an itemset (aub) is defined as the sum of the transaction maximum utilities of transactions. An itemset $\mathrm{X}$ is called a high average-utility upper bound itemset if its average-utility upper-bound is not less than the minimum average-utility count. The HAUI-Miner algorithm scans the database twice to calculate the average-utilities of candidate itemsets. During the first database scan, the set of high average-utilityupper-bound 1-itemsets are retrieved [12]. Then AUlists of 1-itemsets are constructed. During the second database scan, unpromising itemsets i.e whose aub less than the minimum average-utility count are removed. Then the database is revised by removing all unpromising items. Now utility values are calculated using AU list structure.

\subsection{Efficient high average-utility pattern mining (EHAUPM) algorithm}

The algorithms proposed so far uses AUUB model to overestimate the average-utilities of itemsets. To improve the performance of HAUI miner, Lin et al. [12] proposed two novel tighter upper-bound models. The looser upper-bound model (LUB) considers the remaining-maximum utility in transactions to reduce the upper-bound on the utilities of itemsets. The second upper-bound model ignores irrelevant items in transactions to further tighten the upper-bound. Three pruning strategies are also implemented to reduce the search space. The AUUB value is greatly affected by high utility items in a transaction. However, this value is a very loose upper-bound on the average-utility of item. Hence, the item may not be high average items during the mining process. Hence two novel upper-bounds are proposed to reduce the number of unpromising itemsets. A modified average-utility (MAU)-list structure is developed to avoid performing multiple database scans and keeps the required information in memory. The search space of the designed algorithm for mining high average patterns can be represented as an enumeration tree $[10,12]$. If the $l u b(X)$ value of an itemset $\mathrm{X}$ is less than the minimum high average utility count, any extension $\mathrm{Y}$ of $\mathrm{X}$ is not a HAUI. Hence, all extensions of the itemset $\mathrm{X}$ can be ignored while ensuring that all HAUIs can still be found, thus preserving the completeness and correctness of the designed algorithm.

\section{Experimental results}

All algorithms were implemented in Java and experiments were carried on a computer having an Intel(R) Core $3.60 \mathrm{GHz}$ processor with $8 \mathrm{~GB}$ of main memory. The algorithms are compared using RETAIL and CHESS datasets. Here execution time, number of itemsets retrieved and memory space are taken as performance measures. 


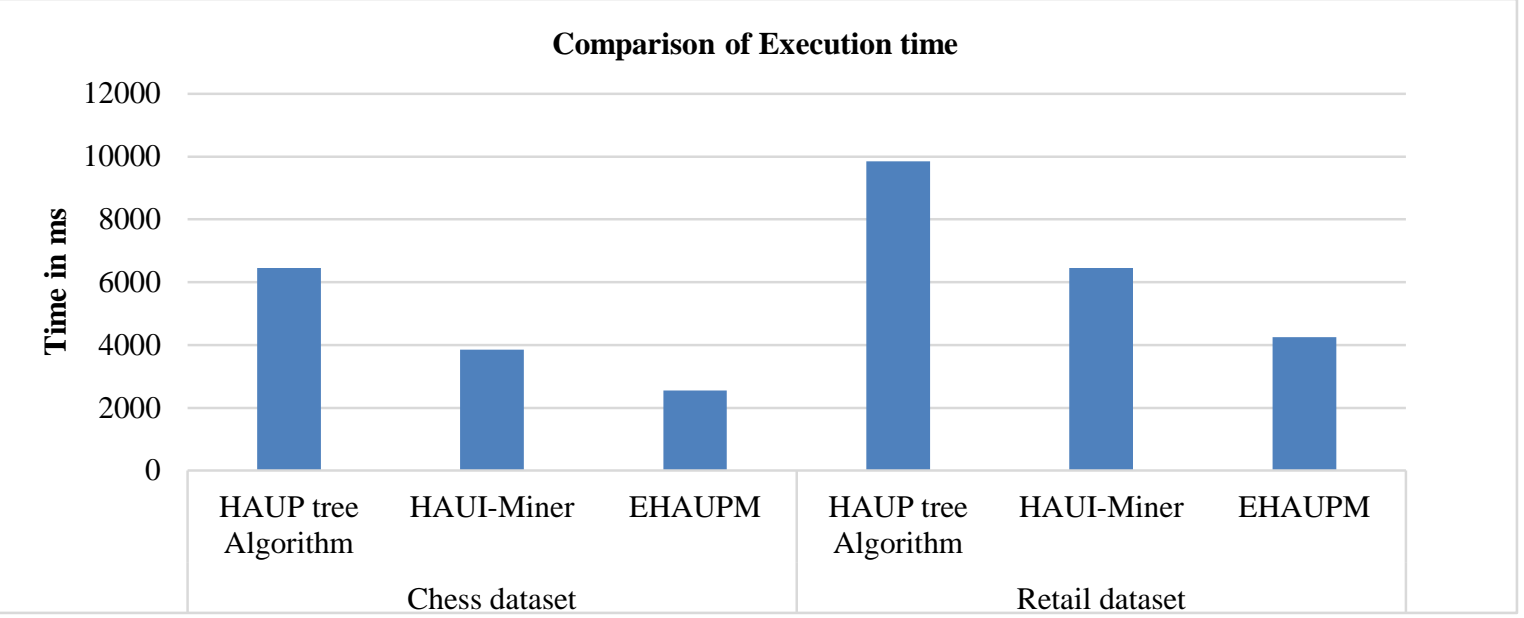

Figure 1 Comparison of execution time in CHESS and RETAIL dataset

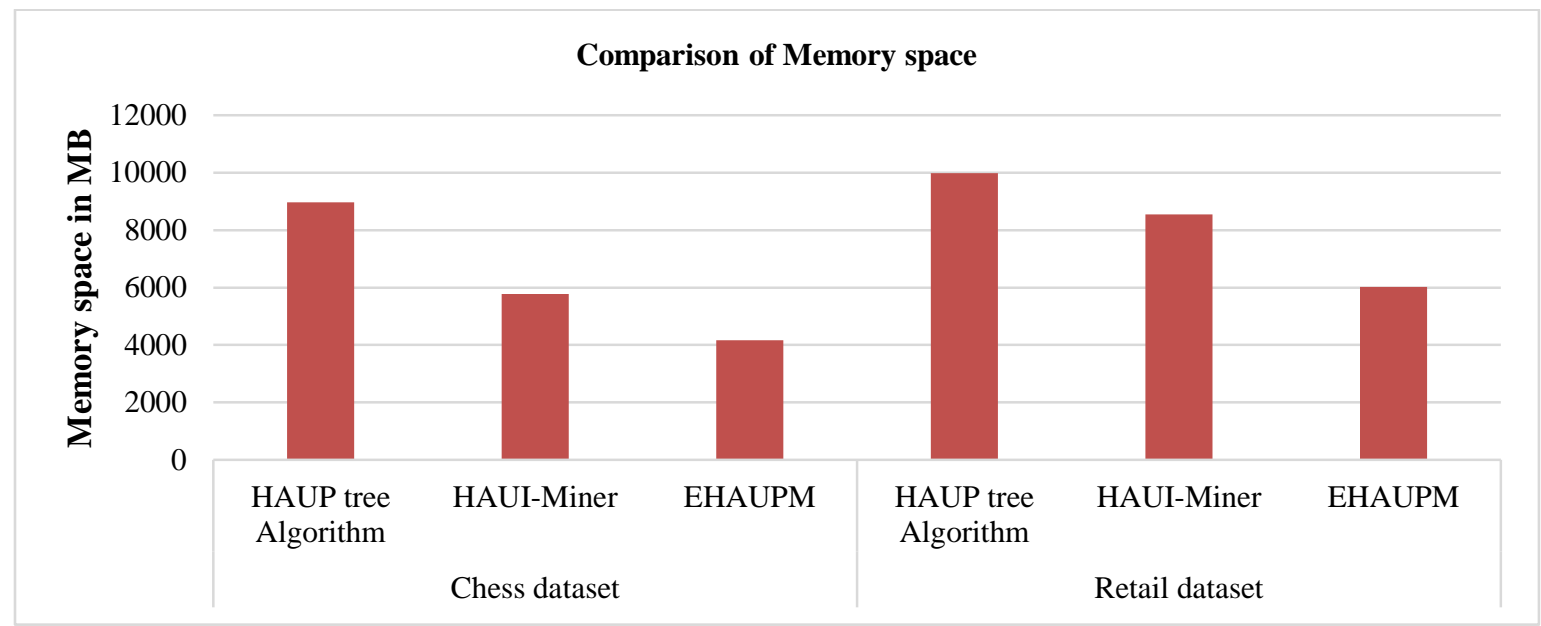

Figure 2 Comparison of memory space in CHESS and RETAIL dataset

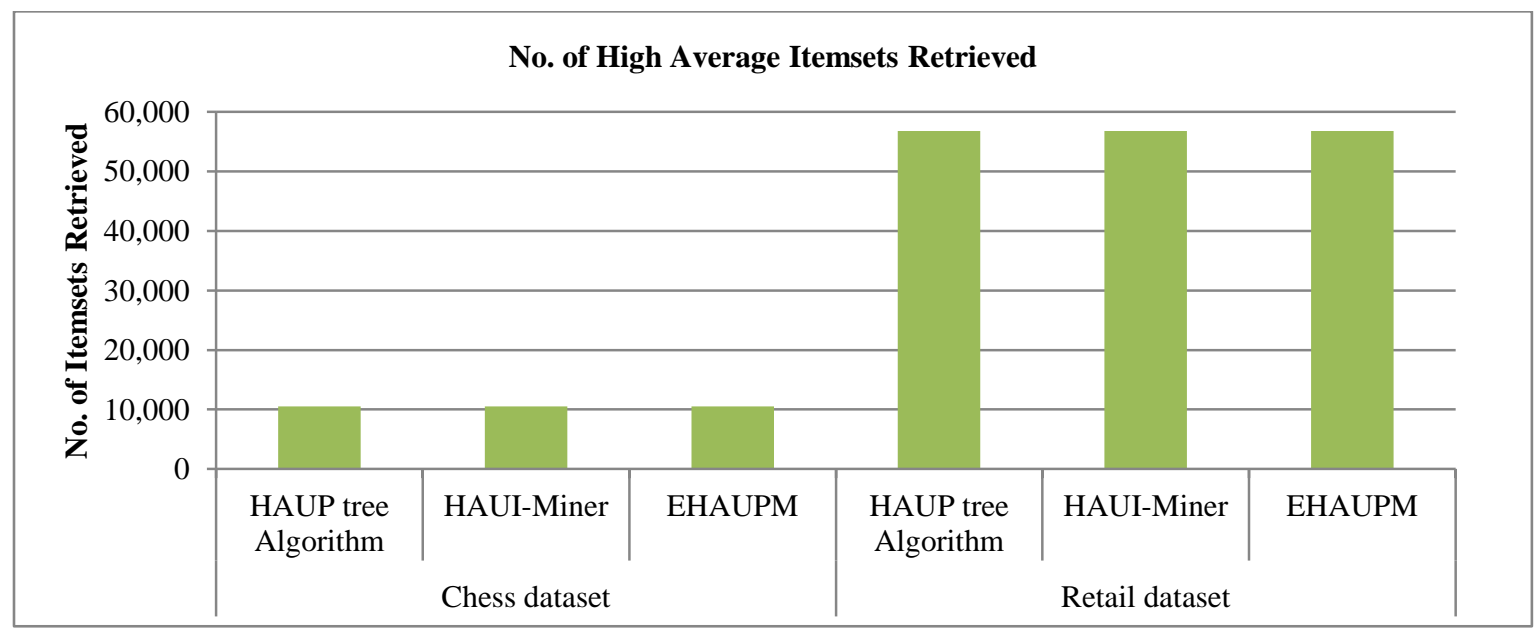

Figure 3 Comparison of number of itemsets retrieved in CHESS and RETAIL dataset 
From the Figures 1-3, it was found that EHAUPM was efficient in terms of execution time and memory space, when compared with other existing algorithms. It consumes less memory space and executes firstly than the remaining ones.

\section{Conclusion}

Traditional high-utility itemset mining considers only the significant utility values of items. Since the utility of larger itemset is greater than the utility of smaller itemset, traditional utility mining algorithms suffer some drawbacks. Thus, the traditional utility measure is not a fair measurement in real world applications. To address this issue, the problem of high average-utility itemset mining has been proposed. It has attracted a lot of attention since it provides a useful alternative interestingness measure to evaluate the discovered patterns. In this paper HAUP tree Algorithm, HAUI-Miner and EHAUPM algorithms are compared and their performances are measured. From the result it was found that, EHAUPM performs better.

\section{Acknowledgment}

None.

\section{Conflicts of interest}

The authors have no conflicts of interest to declare.

\section{References}

[1] Agrawal R, Imieliński T, Swami A. Mining association rules between sets of items in large databases. In ACM SIGMOD record 1993 (pp. 20716). ACM.

[2] Ahmed CF, Tanbeer SK, Jeong BS, Lee YK. Efficient tree structures for high utility pattern mining in incremental databases. IEEE Transactions on Knowledge and Data Engineering. 2009; 21(12):170821

[3] Krishnamoorthy S. Pruning strategies for mining high utility itemsets. Expert Systems with Applications. 2015; 42(5):2371-81.

[4] Sivamathi C, Vijayarani S. Performance analysis of utility mining algorithms. In international conference on inventive computation technologies 2016 (pp. 1-4). IEEE.

[5] Lin JC, Li T, Fournier-Viger P, Hong TP, Su JH. Efficient mining of high average-utility itemsets with multiple minimum thresholds. In industrial conference on data mining 2016 (pp. 14-28). Springer, Cham.

[6] Fournier-Viger P, Wu CW, Zida S, Tseng VS. FHM: faster high-utility itemset mining using estimated utility co-occurrence pruning. In international symposium on methodologies for intelligent systems 2014 (pp. 83-92). Springer, Cham.

[7] Lan GC, Hong TP, Tseng VS. Efficiently mining high average-utility itemsets with an improved upper- bound strategy. International Journal of Information Technology \& Decision Making. 2012; 11(5):100930.

[8] Han J, Pei J, Yin Y, Mao R. Mining frequent patterns without candidate generation: a frequent-pattern tree approach. Data Mining and Knowledge Discovery. 2004; 8(1):53-87.

[9] Hong TP, Lee CH, Wang SL. Mining high averageutility itemsets. In international conference on systems, man and cybernetics 2009 (pp. 2526-30). IEEE.

[10] Lu T, Vo B, Nguyen HT, Hong TP. A new method for mining high average utility itemsets. In IFIP international conference on computer information systems and industrial management 2014 (pp. 33-42). Springer, Berlin, Heidelberg.

[11] Lin JC, Li T, Fournier-Viger P, Hong TP, Zhan J, Voznak M. An efficient algorithm to mine high average-utility itemsets. Advanced Engineering Informatics. 2016; 30(2):233-43.

[12] Lin CW, Hong TP, Lu WH. Efficiently mining high average utility itemsets with a tree structure. In Asian conference on intelligent information and database systems 2010 (pp. 131-9). Springer, Berlin, Heidelberg.

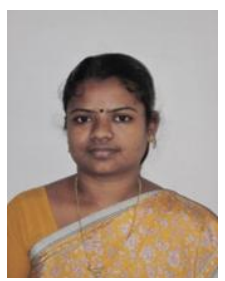

C. Sivamathi Abarajithan is pursuing Ph.D in the Department of Computer Science, Bharathiar University, Coimbatore, India. She has Completed Master of Science in Computer Science and Information Technology. She completed Master of Philosophy in Computer Science. Her research areas include Data Mining, Utility Mining, Data Streams and Big Data. She has five years of teaching experience. She has published papers in international journals. She has attend and presented papers in national and international conferences.

Email: c.sivamathi@gmail.com

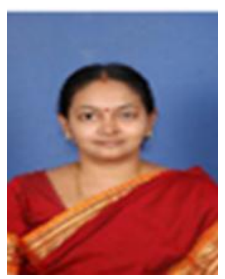

S.Vijayarani Mohan is an Assistant Professor of Department of Computer Science at Bharathiar University, Coimbatore, India. She has obtained M.C.A., M.Phil., and Ph.D., in Computer Science. She has 10 years of teaching/research and 10 years of technical experience. Her research interests include Data Mining, Privacy Issues in Data Mining, Text Mining, Web Mining, Data Streams and Information Retrieval. She has published more than 80 research articles in national/international journals. She also presented research papers in international/national conferences. She has authored a book and guided more than 25 research scholars. She is a life member in professional bodies like CSI, ISCA, IAENG, IRED and UACEE. 